Collectif, La Lotharingie en question : identités, oppositions, intégration / Lotharingische Identitäten im Spannungsfeld: zwischen integrativen und partikularen Kräften, actes des $14^{\text {es }}$ Journées lotharingiennes, 10-13 octobre 2006

\title{
Nicolas Ruffini-Ronzani
}

\section{OpenEdition}

\section{Journals}

Édition électronique

URL : https://journals.openedition.org/ccm/7518

DOI : $10.4000 / \mathrm{ccm} .7518$

ISSN : 2119-1026

Éditeur

Centre d'études supérieures de civilisation médiévale/Université de Poitiers

Édition imprimée

Date de publication : 1 juin 2021

Pagination : 177-180

ISBN : 978-2-490783-09-0

ISSN : 0007-9731

\section{Référence électronique}

Nicolas Ruffini-Ronzani, « Collectif, La Lotharingie en question : identités, oppositions,

intégration / Lotharingische Identitäten im Spannungsfeld: zwischen integrativen und partikularen Kräften, actes des 14es Journées lotharingiennes, 10-13 octobre 2006 », Cahiers de civilisation médiévale [En ligne], 254 | 2021, mis en ligne le 01 juin 2021, consulté le 30 novembre 2022. URL : http:// journals.openedition.org/ccm/7518 ; DOI : https://doi.org/10.4000/ccm.7518

\section{(c) (i) $\odot$}

Creative Commons - Attribution - Pas d'Utilisation Commerciale - Pas de Modification 4.0 International - CC BY-NC-ND 4.0

https://creativecommons.org/licenses/by-nc-nd/4.0/ 
La Lotharingie en question : identités, oppositions, intégration / Lotharingische Identitäten im Spannungsfeld: zwischen integrativen und partikularen Kräften, actes des $14^{\text {es }}$ Journées lotharingiennes, 10-13 octobre 2006, Michel Margue et Hérold Pettiau (dir.), Luxembourg, Section historique de l'Institut Grand-Ducal (Publications de la section historique de l'Institut Grand-Ducal de Luxembourg, 126 / Publications du CLUDEM, 26), 2018.

La parution des actes de Journées lotharingiennes constitue toujours un moment attendu, tant les ouvrages de cette collection nous ont accoutumés à rassembler en un même volume des contributions cohérentes et de haute volée autour d'un espace bien balisé - que l'on songe, par ex., à la publication précédente sur La forêt en Lotharingie médiévale. Par leur qualité, les actes des quatorzièmes Journées lotharingiennes ne dérogent pas à la règle, même si l'on regrettera qu'ils paraissent avec un retard considérable. Les historiens étant fils de leur temps, la rencontre dont les dix-huit interventions sont ici publiées se consacrait à un sujet qui, depuis le milieu des années 2000 , se trouve au cour de débats aux relents trop souvent nauséabonds : celui des identités. Afin d'éviter des écueils dangereux, les éditeurs ont heureusement fait le choix de ne pas envisager la question sous l'angle essentialiste, mais de privilégier l'étude des processus de constructions identitaires au sein de l'espace lotharingien. Une telle démarche impliquait de relire les sources avec l'objectif d'y déceler des traces témoignant de la formation d'identités nationales, régionales ou sous-régionales au sein d'une entité politique dont l'existence a, somme toute, été assez éphémère. Le défi était grand, le pari pas gagné d'avance, mais les conclusions de nombreux articles s'avèrent pleinement satisfaisantes.

Le livre s'ouvre sur une réflexion liminaire de JeanMarie Moeglin, dont les propos ne concernent pas spécifiquement l'espace lotharingien, mais posent la question des identités « nationales » durant la seconde moitié du Moyen Âge. Affirmant, à raison, que « les identités sont d'abord des constructions dans lesquelles l'imaginaire produit de la réalité » (p. 16), J.-M. Moeglin précise que, pour prendre corps, ces dernières doivent s'articuler autour de référentiels communs. Pour la période médiévale du moins, ces référentiels sont généralement définis par les élites (aristocratiques, urbaines ou ecclésiastiques). La compréhension de ce processus complexe implique d'identifier un certain nombre de vecteurs identitaires. L'hagiographie constitue l'un de ceux-ci à l'époque médiévale. S'appuyant sur des sources liturgiques, et plus spécifiquement sur des calendriers et des martyrologes, Thomas Bauer conclut à l'existence de saints aux cultes spécifiquement lotharingiens. Dans une même veine, mais en bornant son champ de recherche à l'évêché de Metz, Anne Wagner met en relief le rôle des reliques utilisées par les prélats messins dans le cadre d'une politique d'appropriation du territoire diocésain. En prenant pour point de départ de sa réflexion une curieuse liste royale présente dans le Liber Memorialis de Remiremont (années 860), Michèle Gaillard démontre que les Lotharingiens ne se sont jamais revendiqués d'un héritage austrasien avant le $\mathrm{XI}^{\mathrm{e}} \mathrm{s}$., mais qu'ils ont plutôt cherché à s'inscrire dans la continuité d'un passé pippinide et carolingien. Soulignant que les vecteurs identitaires médiévaux parvenus jusqu'à nous ne sont pas uniquement des écrits, mais aussi des traces matérielles, Elizabeth Den Hartog établit 
que la chapelle royale d'Aix-la-Chapelle a servi de modèle à de nombreux édifices. Ces imitations sont particulièrement nombreuses en Basse-Lotharingie au tournant des $\mathrm{X}^{\mathrm{e}}$ et $\mathrm{XI}^{\mathrm{e}} \mathrm{s}$. (église Saint-Jean l'Évangéliste de Liège, chapelle Saint-Nicolas de Nimègue, etc.). Il semblerait que des proches du pouvoir ottonien soient le plus souvent à l'initiative de ces constructions, lesquelles ont évidemment une forte valeur symbolique en raison du prestige de la chapelle impériale.

La question de l'existence d'identités locales au sein de l'espace lotharingien est soulevée par JeanLouis Kupper et Michel de Waha. Le premier déduit des sources que les Liégeois des $\mathrm{X}^{\mathrm{e}}-\mathrm{XII}^{\mathrm{e}} \mathrm{s}$. - tant les clercs que les grands laïcs - avaient conscience d'appartenir à deux « patries » dont la première était enchâssée dans la seconde : le diocèse de Liège et le duché de Basse-Lotharingie. Analysant ensuite les rapports de rivalité qui opposent l'évêque au duc, J.-L. Kupper révèle combien la paix de Dieu liégeoise de 1081 a contribué à accroître le pouvoir du prélat au détriment du prince. Au terme d'une longue démonstration, M. de Waha constate, pour sa part, qu'au sein du Hainaut et du Brabant « des formes d'identités régionales propres se manifestant au niveau du pagus $\mathrm{s}^{\prime}$ imposent dès le $\mathrm{X}^{\mathrm{e}} \mathrm{s}$. au plus tard et se maintiennent jusqu'à la fin du $\mathrm{XII}^{\mathrm{e}}$ siècle au moins » (p. 275). En Hainaut, le cas est patent autour de Valenciennes, où l'on voit se développer des particularismes très forts - j'ajoute que ceux-ci se maintiendront longtemps, parfois même jusqu'à la fin du Moyen Âge. Ces identités « sous-régionales » ne sont cependant pas exclusives d'un sentiment d'appartenance à des entités plus vastes. Hier comme aujourd'hui, les identités multiples sont en effet susceptibles de s'emboîter les unes dans les autres, les acteurs présents sur la scène politique jouant plutôt de l'une ou plutôt de l'autre en fonction de leurs intérêts du moment.

S'il allait de soi de s'interroger sur la définition que les Lotharingiens donnaient de leur propre identité, il était également indispensable de déterminer comment la Lotharingie était perçue par ses voisins. C'est ce qu'entreprennent Sophie Glansdorff et Charles West. Envisageant une période courant de la disparition

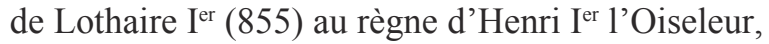
la première démontre que, dès le milieu du $\mathrm{IX}^{\mathrm{e}} \mathrm{s}$., la Lotharingie a occupé une place majeure dans le programme politique des rois de Francie orientale. En dehors de la sphère royale, de forts liens d'amitié paraissent d'ailleurs se tisser entre les évêques franc-orientaux et leurs homologues lotharingiens à partir de 870. Les troubles des règnes de Zwentibold et de Louis l'Enfant distendront néanmoins les relations au tournant des $\mathrm{IX}^{\mathrm{e}}$ et $\mathrm{X}^{\mathrm{e}} \mathrm{S}$., en contribuant à l'émergence de particularismes. C. West ne formule pas des conclusions radicalement différentes à propos de la Francie occidentale. Selon lui, la Lotharingie exerce également un attrait indéniable sur les souverains francs occidentaux, ne serait-ce que parce qu'elle a été le berceau de l'Empire carolingien. Il souligne en outre que les échanges culturels et intellectuels entre l'espace lotharingien et la Francie occidentale ont probablement été plus nombreux que ne le laissent à penser les sources.

La question de l'identité est évidemment liée au sentiment d'appartenance à un territoire. En toute logique, plusieurs articles abordent donc la problématique de l'espace et du territoire, laquelle a été au centre de débats historiographiques récents. Les actes du colloque étant publiés avec retard, ils reflètent un état de la recherche antérieur à la parution de contributions récentes et majeures. C'est regrettable, car l'on aurait été curieux de lire les prises de position des auteurs sur certaines productions des années 2010 - on songe, en particulier, aux travaux de Florian Mazel sur la territorialisation de l'espace et à la thèse de Jens Schneider consacrée à la Lotharingie (Florian MAzel, L'évêque et le territoire: l'invention médiévale de l'espace, Paris, Seuil [L'Univers historique], 2016; Jens SCHNEIDER, Auf der Suche nach dem verlorenen Reich: Lotharingien im 9. und 10. Jahrhundert, Régine LE JAN (préf.), Cologne, Böhlau [Publications du Centre luxembourgeois de documentation et d'études médiévales, 30], 2010). Dans sa contribution, J. Schneider pose les bases théoriques et méthodologiques d'une étude de l'espace lotharingien. Il est sans doute préférable aujourd'hui de se référer au livre issu de sa thèse, puisque l'a. y développe plus amplement ses vues. Revenant sur un espace qui lui est cher, Michel Parisse dresse une rapide synthèse de l'histoire des frontières de la Lorraine du $\mathrm{IX}^{\mathrm{e}}$ au XIII ${ }^{\mathrm{e}} \mathrm{s}$. Il y insiste notamment sur l'accroissement de l'influence française dans la région à partir du XII ${ }^{e} \mathrm{~s}$. Dans une longue et belle enquête, Hérold Pettiau retrace les itinéraires et les modalités de présence des souverains francs occidentaux et orientaux dans l'espace lotharingien entre le décès de Lothaire II (869) et l'avènement d'Otton I Ir (936). 
Il met en évidence l'existence de plusieurs pôles de fréquentation, dont la liste évolue quelque peu au fil du temps, même si Aix-la-Chapelle occupera toujours une position prééminente. Ainsi, alors que jusqu'en 895, Metz constitue le second pôle régional d'importance avec Aix, le roi Zwentibold semble se replier sur Trèves entre 895 et 900 , probablement parce qu'il n'a pas d'autre choix, son réseau de pouvoir étant plus faible et moins étendu. Metz ne retrouvera pas son lustre d'antan sous les premiers Ottoniens, puisque ceux-ci privilégieront les sites d'Aix, de Nimègue et de Cologne.

D'autres articles s'inscrivent dans une perspective plus traditionnelle. La contribution magistrale de M. Margue envisage les destins croisés de deux figures importantes mais très largement dépréciées de l'histoire de la Lotharingie : celles du roi Zwentibold (895-900) et du duc Gislebert (928-939). Il n'est guère possible de synthétiser en quelques lignes les apports de cette très longue étude (près de $100 \mathrm{p}$.!), qui vient d'une certaine manière prolonger des réflexions déjà formulées par le passé (voir Alain Dierkens et Michel Margue, « Memoria ou damnatio memoriae? L'image de Gislebert, duc de Lotharingie [ $\dagger$ 939]», dans Retour aux sources : textes, études et documents d'histoire médiévale offerts à Michel Parisse, Sylvain Gouguenheim et Monique Goullet [éd.], Paris, Picard, 2004, p. 869-890). Désamorçant les nombreux pièges tendus par des sources d'une interprétation difficile, M. Margue s'efforce, dans un premier temps, de mieux cerner les contours des politiques de Zwentibold, qui semble avoir vainement tenté de constituer un royaume lotharingien autonome, et de Gislebert, qui a souhaité reproduire à l'échelle ducale le modèle royal carolingien, avant que la reprise en main ottonienne ne le mette en échec. Dans un second temps, M. Margue évoque la manière dont l'historiographie du $\mathrm{x}^{\mathrm{e}} \mathrm{s}$. a contribué à forger une image globalement négative de ces personnages dont la "légende noire » s'est transmise jusqu'à nous. Dans une très belle enquête, Arnoud-Jan A. Bijsterveld s'intéresse à la trajectoire d'un lignage lotharingien mal connu, les Rode. Au-delà de son intérêt évident pour l'histoire politique régionale, cette étude retient l'attention par ses fondements théoriques. S'inspirant de la sociologie politique anglo-saxonne (et plus particulièrement des travaux de Michael ManN, The Sources of Social Power, I: A History of Power from the Beginning to A.D. 1760, Cambridge, Cambridge University Press, 1986), l'a. définit les éléments constituant les fondements du pouvoir aristocratique au Moyen Âge central. Le modèle ainsi bâti pourrait être appliqué à d'autres dossiers.

Enfin, un dernier ensemble d'articles porte sur la persistance et les formes prises par le concept de «Lotharingie » des derniers siècles du Moyen Âge jusqu'au lendemain de la seconde guerre mondiale. Les contributeurs démontrent que si l'idée lotharingienne ne s'est jamais totalement effacée et a régulièrement été réactivée pour répondre à des ambitions politiques, elle s'est progressivement vidée de sa substance. C'est probablement au cour de la BasseLotharingie, dans le Brabant de David Guilardian, que les évolutions de la notion sont les plus intrigantes. En effet, alors même que leurs ancêtres ont longtemps lutté pour s'assurer la dignité de duc de Basse-Lotharingie, les princes brabançons des $\mathrm{XIII}^{\mathrm{e}}-\mathrm{XV}^{\mathrm{e}} \mathrm{s}$. jouent finalement assez peu de ce titre dans leurs titulatures et dans les œuvres narratives produites sous leur autorité. À l'idée lotharingienne, ils privilégient principalement le concept de Brabant, lequel leur est apparu comme un vecteur identitaire plus puissant dans la construction de leur principauté. Succédant aux princes brabançons en 1430, les ducs de Bourgogne n'ont pas non plus cherché à promouvoir une identité lotharingienne dans leur immense ensemble territorial, comme l'affirme Robert Stein. Aux yeux de l'historien néerlandais, les terres gouvernées par les Bourguignons formaient d'ailleurs un ensemble hétérogène dont la fracture n'était pas Nord-Sud, comme cela a longtemps été soutenu, mais Est-Ouest. Les Temps modernes et l'époque contemporaine sont également à quelques reprises le théâtre d'une réactivation du souvenir lotharingien, comme l'explique François Pernot. Les nouvelles « Lotharingie » imaginées par les gouvernants sont alors souvent des constructions de l'esprit sans grand lien avec l'entité du haut Moyen Âge, des projets confus visant à la création d'un «État-tampon » dans des contextes politiques troublés. Tout bien pesé, c'est peut-être dans la littérature épique des $\mathrm{XII}^{\mathrm{e}}-\mathrm{XIII}{ }^{\mathrm{e}} \mathrm{s}$. que l'imaginaire lotharingien est resté le plus prégnant, comme le note François Walter à partir, notamment, de l'exemple de la Geste des Lorrains.

En définitive, le volume édité par M. Margue et $\mathrm{H}$. Pettiau constitue donc une réussite dont de belles contributions feront date dans les études 
lotharingiennes. Néanmoins, on regrettera, d'une part, l'absence d'une conclusion qui aurait permis de faire la synthèse de ces riches travaux et, d'autre part, la publication tardive de ces actes de colloque. Les articles rassemblés témoignent d'un état de l'historiographie vieux de plusieurs années, même si l'un ou l'autre auteur a eu l'heureuse initiative de faire apparaître des compléments bibliographiques en fin de texte. Par la force des choses, il en résulte que des ouvrages récents et importants n'ont pas été pris en compte par les contributeurs. Les lacunes sont plus ou moins prononcées selon les thèmes abordés - c'est sans doute autour de la problématique de l'espace et du territoire qu'elles sont les plus nombreuses. Il importe que le lecteur en soit conscient à l'heure d'ouvrir La Lotharingie en question, qui, malgré cette faiblesse, reste un ouvrage dont il y aura beaucoup à tirer.

Nicolas RuFfINI-RonZANI Pratiques médiévales de l'écrit - PraME Université de Namur 